\title{
Endoscopic clipping of intraventricular aneurysms using the "wet-field" technique
}

\author{
Kazuhito Takeuchi, MD, PhD, ${ }^{1}$ Takashi Handa, MD, PhD, ${ }^{2}$ Jonsu Chu, MD, ${ }^{1}$ Kentaro Wada, MD, ${ }^{1}$ and \\ Toshihiko Wakabayashi, MD, PhD'
}

\begin{abstract}
1Department of Neurosurgery, Nagoya University Graduate School of Medicine, Nagoya City, Aichi; and 2Department of Neurosurgery, Tokoname Municipal Hospital, Tokoname City, Aichi, Japan
\end{abstract}

\begin{abstract}
Intraventricular hemorrhage and intracerebral aneurysms are relatively frequent complications associated with moyamoya disease. Prevention of aneurysm rerupture is important because it significantly decreases the morbidity and mortality rates. Aneurysms arising distal to collateral flow are sometimes observed in patients with intraventricular hemorrhage; however, the treatment of these aneurysms remains challenging because of their deep-seated location in the brain and accompanying narrow surgical corridor. The authors describe a neuroendoscopic aneurysm clipping technique performed in 2 cases using a small-diameter tubular retractor for intraventricular aneurysms of the distal lateral posterior choroidal artery. In this technique, the surgical field was continuously irrigated with artificial CSF to keep the ventricle size intact, and aneurysm clipping was performed through a tubular retractor that was introduced with neuronavigational guidance. The patients' postoperative courses were uneventful, and CT angiography revealed complete clipping of the aneurysms and patent parent arteries. Endoscopic clipping using a tubular retractor is an effective and less invasive alternative for treating intraventricular aneurysms. The wet-field endoscopic technique is performed in an aqueous field and maintains an intact ventricle size, allowing for a clear surgical view and a wider, enhanced surgical field.
\end{abstract}

https://thejns.org/doi/abs/10.3171/2018.1.JNS172393

KEYWORDS aneurysm; clipping; moyamoya disease; endoscope; intraventricular hemorrhage; port surgery; surgical technique; vascular disorders

I NTRACRANIAL hemorrhage is often observed in adult patients with moyamoya disease. Hemodynamic stress on the frail moyamoya vessels is considered to be the main cause of hemorrhage. Some patients develop aneurysms in the major and peripheral arteries, and the prognosis of patients with rebleeding is poor., ${ }^{6,13}$ Therefore, aggressive surgical treatment is recommended when the cause of bleeding can be accurately determined..$^{13}$

The reported treatment strategies for intraventricular aneurysms associated with moyamoya disease are as follows: conservative therapy with revascularization surgery; direct surgical intervention, including clipping, resection, and trapping; and endovascular embolization., ${ }^{1,5,8,9}$ Each individual case should be meticulously considered when choosing the treatment strategy.

In this technical report, we performed endoscopic clipping in 2 cases as the main treatment for intraventricular aneurysms. The procedures were performed using artificial CSF, and therefore, we refer to this surgery as the "wet-field" technique.

\section{Case Reports \\ Case 1}

A 37-year-old man presented to the emergency department with a headache and vomiting. The patient's past medical history included intracranial hemorrhage and a prior diagnosis of moyamoya disease. Brain CT revealed intraventricular hemorrhage (Fig. 1). Because the patient's neurological signs were not severe and he did not have any apparent clinical or radiographic evidence of hydrocephalus, conservative therapy was initially chosen, and subsequently, his symptoms resolved within 1 week of admission. Three-dimensional CT angiography demonstrated 

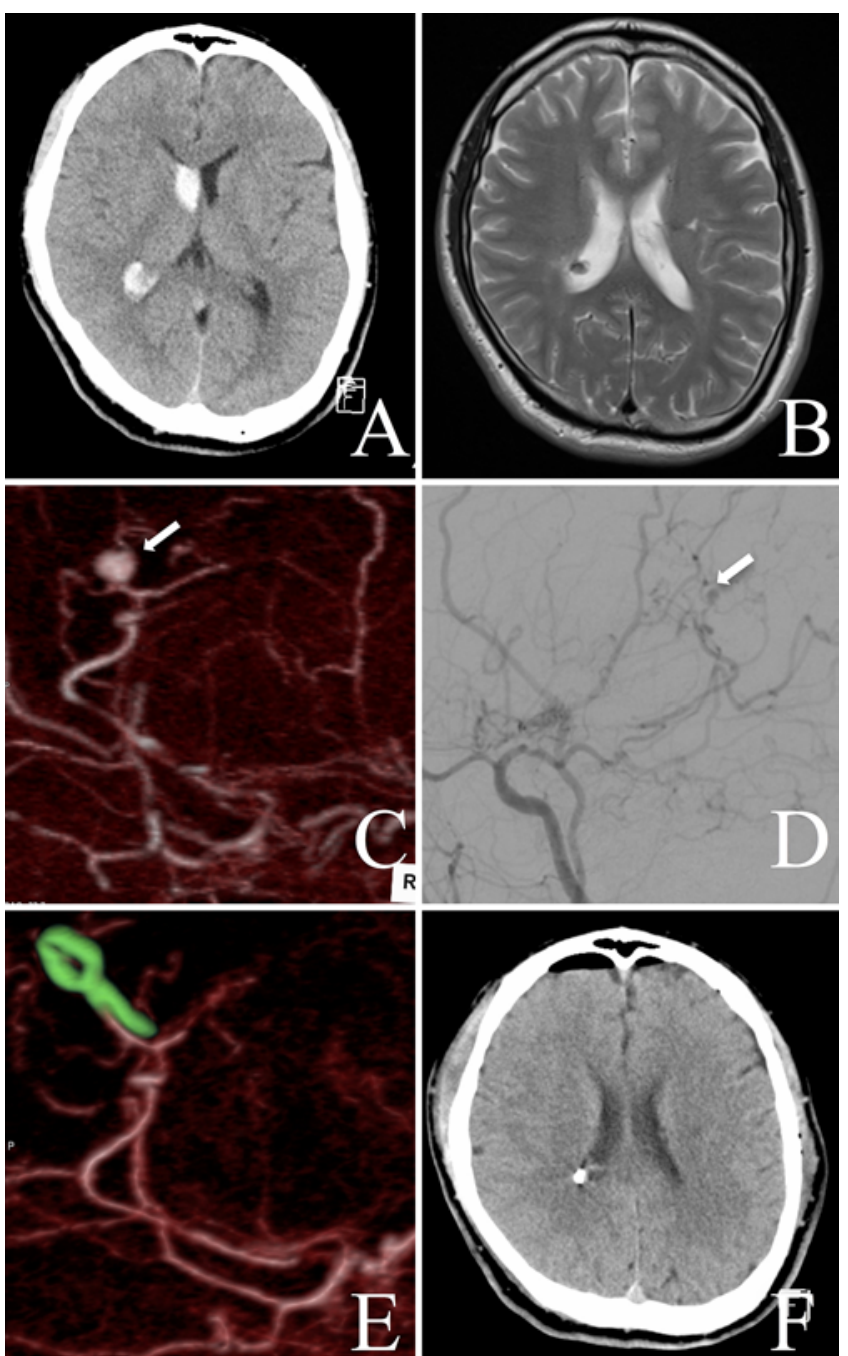

FIG. 1. Case 1. A: Axial CT scan showing a right intraventricular hemorrhage. B: Axial T2-weighted MR image showing an intraventricular aneurysm on the lateral wall of the right lateral ventricle. C: Threedimensional CT angiogram showing a saccular aneurysm (white arrow) arising from the bifurcation of the distal lateral posterior choroidal artery. D: Internal carotid artery angiogram (lateral view) showing a saccular aneurysm (white arrow) with tortuous and narrow parent arteries.

E: Three-dimensional CT angiogram demonstrating complete aneurysm clipping and a patent parent artery. F: Postoperative CT scan demonstrating no evidence of the hemorrhage or ischemic changes. Figure is available in color online only.

typical findings of proximal internal carotid stenosis and microvascular proliferation in the basal ganglia consistent with moyamoya disease, and a 4-mm-diameter aneurysm was found on the lateral wall of the right lateral ventricle. MRI showed the aneurysm body buried in the subependymal surface of the lateral ventricle. A right cerebral angiogram showed a saccular aneurysm at the bifurcation of the right distal lateral posterior choroidal artery that was postulated to be the cause of bleeding. Because of the distal location of the aneurysm and the tortuous, narrow parent artery, endovascular treatment was considered unsuitable, while the saccular aneurysm shape and location on the
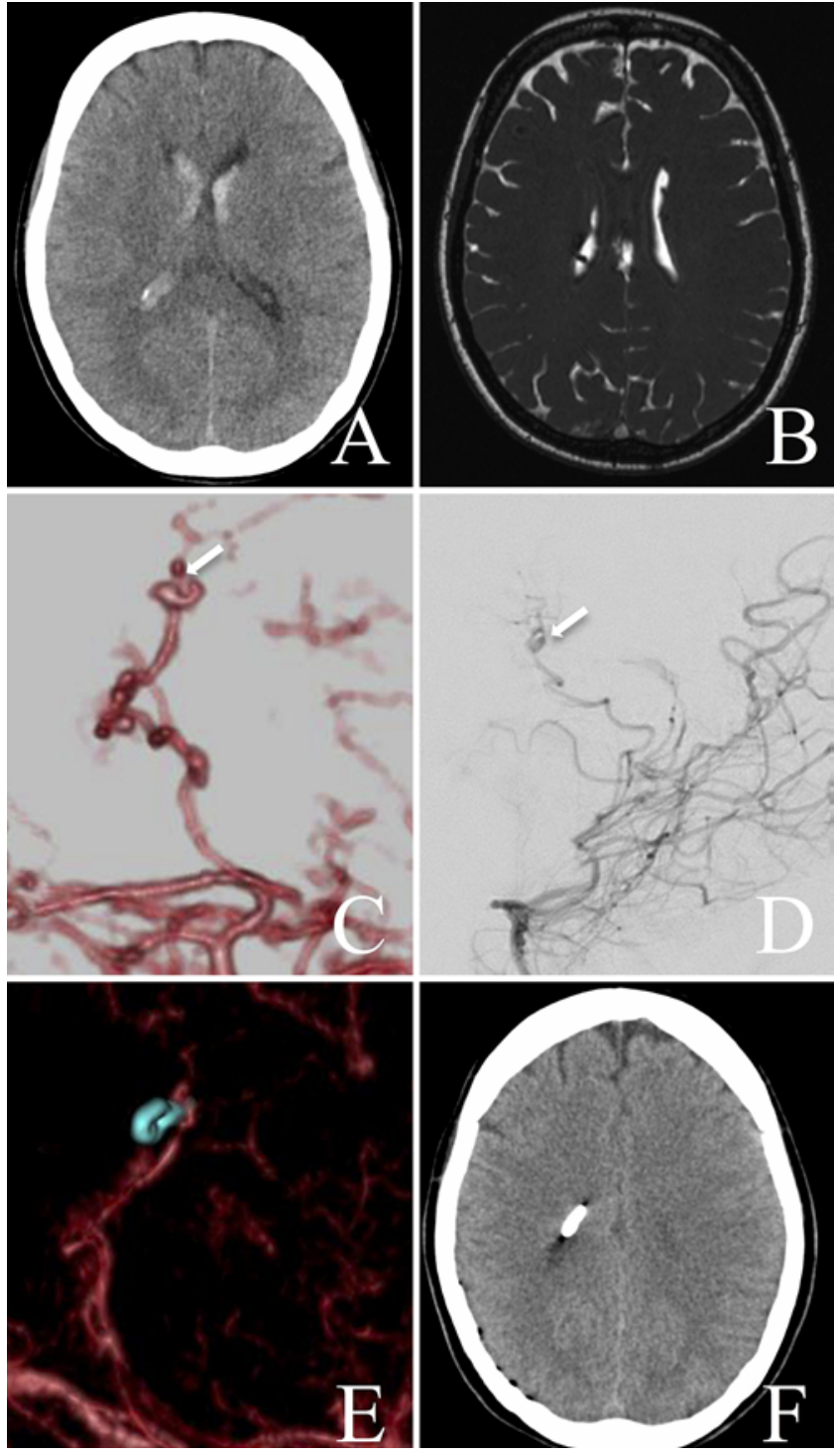

FIG. 2. Case 2. A: Axial CT scan showing intraventricular hemorrhage. B: Axial heavy T2-weighted MR image showing an intraventricular aneurysm on the lateral wall of the right lateral ventricle. C: Threedimensional CT angiogram showing a saccular aneurysm (white arrow) arising from the distal lateral posterior choroidal artery. D: A vertebral artery angiogram (lateral view) showing a saccular aneurysm (white arrows) with tortuous and narrow parent arteries. E: Three-dimensional CT angiogram demonstrating complete aneurysm clipping and a patent parent artery. F: Postoperative CT scan demonstrating no evidence of the hemorrhage or ischemic changes. Figure is available in color online only.

parent artery made it suitable for neck clipping. After informed consent was obtained regarding the various treatment strategies, the patient chose to undergo endoscopic transcylinder intraventricular aneurysm clipping.

\section{Case 2}

A 44-year-old woman presented with the sudden onset of headache. Brain CT revealed an intraventricular hemorrhage without any apparent hydrocephalus (Fig. 2). 

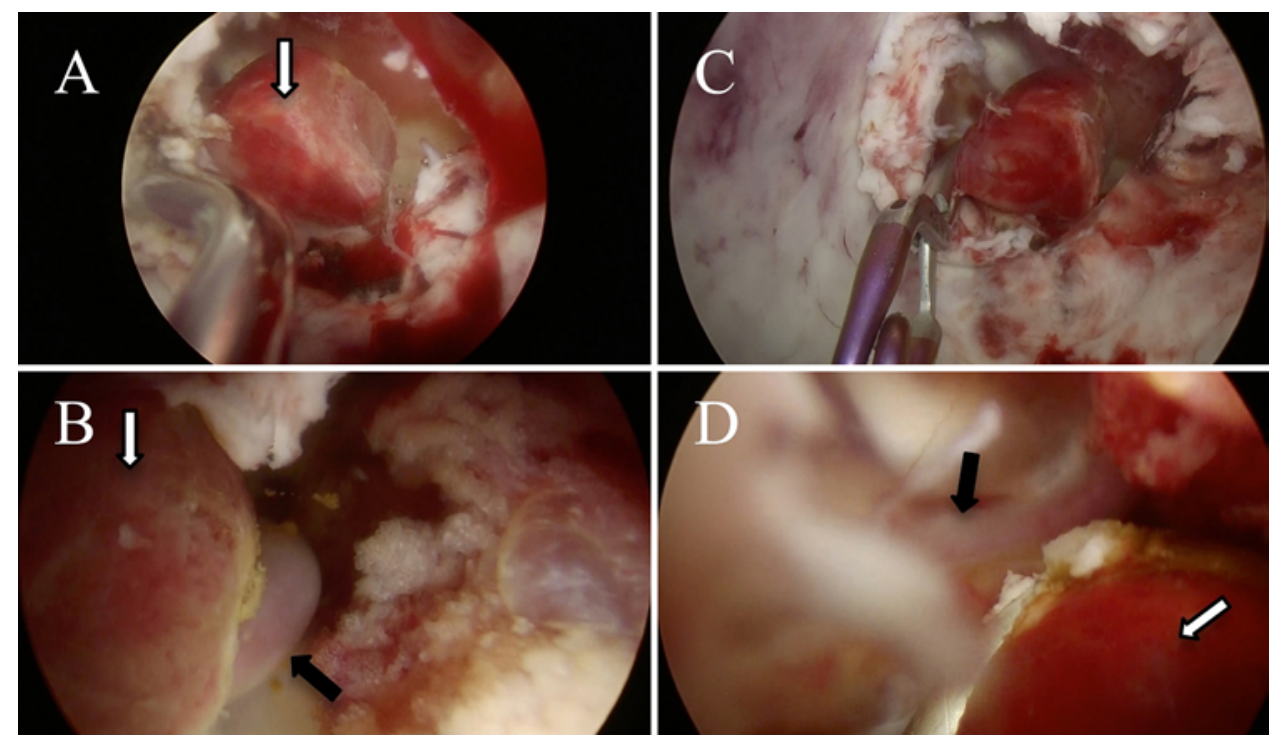

FIG. 3. Case 1. A tubular retractor (12 L [12-mm width $\times 8-\mathrm{mm}$ height $\times 70-\mathrm{mm}$ length] Viewsite Brain Access System, Vycor Medical) was introduced just medial and proximal to the aneurysm inside the right lateral ventricle using neuronavigational guidance. A: The aneurysm is visible on the lateral surface of the wall of the right lateral ventricle (white arrow). B: A $30^{\circ}$ angled endoscope is used to identify the parent artery (black arrow) located behind the aneurysm (white arrow). C: Aneurysmal neck clipping was performed using a single-shaft clip applicator. D: Complete aneurysm clipping (white arrow) and patency of the parent artery (black arrow) are confirmed using $30^{\circ}$ and $70^{\circ}$ degree endoscopes. Figure is available in color online only.

Because the patient's symptoms were mild and she did not have any neurological deficit, conservative therapy was initially chosen. Three-dimensional CT angiography and MRI revealed findings consistent with a diagnosis of moyamoya disease, and a 3-mm-diameter aneurysm was detected on the surface of the lateral wall of the right lateral ventricle. A right cerebral angiogram showed a saccular aneurysm at the bifurcation of the right distal lateral posterior choroidal artery that was postulated to be the cause of bleeding. After informed consent was obtained regarding various treatment strategies, the patient agreed to undergo endoscopic transcylinder clipping of the intraventricular aneurysm.

\section{Surgical Technique}

The patients were placed prone with head fixation using a 4-point Sugita head frame. A 5-cm vertical linear skin incision parallel to the occipital midline followed by a $2.5-\mathrm{cm}$ diameter craniotomy was performed. Using neuronavigational guidance (StealthStation S7, Medtronic), a 6-mm-diameter translucent sheath was inserted in a trajectory toward the lateral ventricle. Next, under endoscopic visualization (Endoarm HD, Olympus), the sheath was positioned just medial to the aneurysm because of the increased risk of rerupture with a direct approach. After reaching the lateral ventricle, the surgical corridor was dilated with an 8.8- $\mathrm{mm}$ translucent sheath, and a tubular retractor system was inserted to ensure a surgical corridor of adequate diameter. The sheath was filled with artificial CSF (Artcereb, Otsuka Pharmaceutical Factory), and most of the subsequent procedures were performed under continuous artificial CSF irrigation. This served to preserve the ventricle size and facilitate exposure of the aneurysm and surrounding arteries without additional retraction. Since the aneurysm was buried in the lateral ventricle ependyma, the body of the aneurysm was carefully dissected away from the ependyma to expose the entire aneurysm and parent vessels. Neck clipping was performed using an angled $30^{\circ}$ rigid endoscope. After applying the clip, complete occlusion of the aneurysm and patency of the parent artery were confirmed using combinations of $30^{\circ}$ and $70^{\circ}$ endoscopic views and intraoperative neuroendoscopic Doppler echo (Figs. 3 and 4, Video 1).

VIDEO 1. Case 1. The patient was placed in the prone position. A small craniotomy was made, and all subsequent procedures were performed under endoscopic visualization. The aneurysm and parent artery were exposed, and a clip was applied to the aneurysm. Complete clipping was confirmed by direct visualization using an angled endoscope and Doppler echo. All endoscopic surgical procedures were performed with continuous aqueous irrigation to maintain adequate ventricle size. Copyright Department of Neurosurgery, Nagoya University. Published with permission. Click here to view.

\section{Postoperative Course}

Postoperatively, neither of the patients demonstrated any neurological deficits, and CT angiography confirmed patent parent arteries with no evidence of residual aneurysms.

\section{Discussion}

The strategy for treating intraventricular aneurysms associated with moyamoya disease remains controversial. ${ }^{13}$ Some patients can be treated with conservative therapy, and spontaneous disappearance after revascularization surgery has been reported. ${ }^{9}$ However, because this out- 


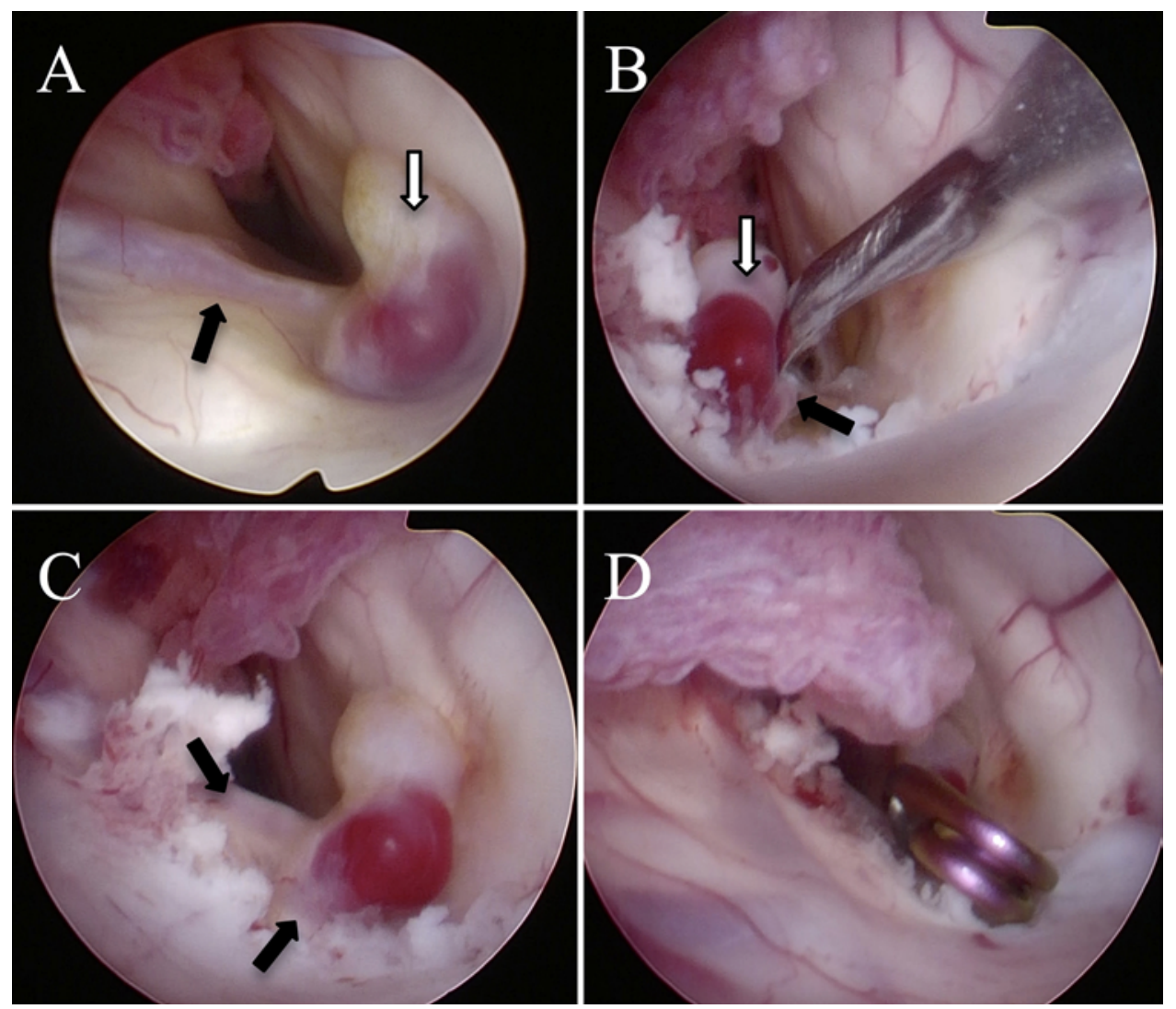

FIG. 4. Case 2. A 10-mm diameter tubular retractor (Neuroport, Hakko Medicals) was introduced just posterior to the aneurysm inside the right lateral ventricle using neuronavigational guidance. A: The aneurysm (white arrow) and parent artery (black arrow) are visible on the cranial surface of the wall of the right lateral ventricle. B: The aneurysm (white arrow) was dissected from the ependyma to expose the aneurysm neck and the distal parent artery (black arrow). C: The aneurysm and the proximal and distal parent artery were exposed. D: Aneurysm neck clipping was performed using a single-shaft clip applicator. Figure is available in color online only.

come is not substantiated in major prospective studies and is consequently not guaranteed, and because aneurysm rerupture can be fatal, adequate surgical intervention is recommended. 7,11

Recently, endovascular treatment for these aneurysms has been popularized as a less invasive treatment strategy. ${ }^{1,48}$ However, the procedure is not without complications, and a successful outcome is largely dependent on the extent of the tortuosity and the diameter of the parent artery. ${ }^{2,14}$ In the present cases, the parent arteries were relatively narrow and tortuous; therefore, endovascular treatment was not thought to be suitable for either case.

Microsurgical treatment is another surgical strategy with several reports of favorable outcomes. ${ }^{3,12,13}$ However, standard microscopic treatment requires a relatively large craniotomy with brain retraction to expose the aneurysm, the parent arteries, and the surrounding delicate neurovascular structures, potentially causing additional ischemic brain damage due to collateral flow disruption and retraction injury. $2,8-10$

The intraventricular vessels, including the parent artery and adjacent veins in patients with moyamoya disease, are a vital part of the normal intracranial hemodynamics, and blood flow should be preserved if at all possible. A tubular retractor system reduces retraction injury to the surrounding surgical corridor. Recently, tubular retractor system-assisted surgeries for intraventricular aneurysms have been reported., ${ }^{2,10}$ In these reports, the aneurysms were well exposed and successfully ligated, and the use of a tubular retractor system reduced the size of the surgical corridor and subsequent risk of retraction injury of the surrounding brain. ${ }^{2,10}$ However, patients in these reports underwent trapping or coagulation of the parent artery, and this can be a cause of ischemia and cerebral infarction. ${ }^{9,10,12}$ In our cases, the aneurysms arose from the bifurcation of the parent arteries, and these were thought to be suitable for clipping surgery with the preservation of the parent arteries.

Furthermore, during standard microscopic surgery, the CSF is typically evacuated to allow visualization of the surgical field, which results in narrowing of the ventricle dimensions. As a consequence, visualization of the surrounding vital vessels can be obscured, leading to an increased risk of injury to the surrounding brain tissue. In the present cases, the patients presented initially with relatively narrow ventricles. Therefore, we applied the wet-field technique to combat intraoperative ventricular narrowing. Continuous artificial CSF irrigation expanded 
the surgical field and kept the surgical field clear. The endoscope provided a clear view of the submerged field and also allowed use of all the standard surgical instruments, even under the "wet-field" condition. Both slow drip irrigation and fast irrigation were used, requiring a 3-way stopcock for the alternation of irrigation speeds. When hemorrhage was detected, rapid irrigation permitted a faster exchange of the artificial CSF and clearer visualization of the bleeding point. A bipolar coagulator could also be used; however, its use is not required because of the relatively rapid, spontaneous hemostasis achieved by the continuous water pressure delivery provided in this wetfield technique.

Although intraventricular aneurysms are rare and surgical interventions are limited, endoscopic transcylinder clipping is a suitable alternative for this kind of deepseated lesion. Furthermore, application of the novel wetfield endoscopic technique provides a relatively wide surgical corridor with enhanced visualization of the surgical field, resulting in a minimally invasive, safe, and effective therapy.

\section{References}

1. Chalouhi N, Tjoumakaris S, Gonzalez LF, Dumont AS, Shah Q, Gordon D, et al: Onyx embolization of a ruptured lenticulostriate artery aneurysm in a patient with moyamoya disease. World Neurosurg 80:436.e7-436.e10, 2013

2. Chen CJ, Caruso J, Starke RM, Ding D, Buell T, Crowley RW, et al: Endoport-assisted microsurgical treatment of a ruptured periventricular aneurysm. Case Rep Neurol Med 2016:8654262, 2016

3. Hamada J, Hashimoto N, Tsukahara T: Moyamoya disease with repeated intraventricular hemorrhage due to aneurysm rupture. Report of two cases. J Neurosurg 80:328-331, 1994

4. Harreld JH, Zomorodi AR: Embolization of an unruptured distal lenticulostriate aneurysm associated with moyamoya disease. AJNR Am J Neuroradiol 32:E42-E43, 2011

5. He K, Zhu W, Chen L, Mao Y: Management of distal choroidal artery aneurysms in patients with moyamoya disease: report of three cases and review of the literature. World J Surg Oncol 11:187, 2013

6. Iwama T, Morimoto M, Hashimoto N, Goto Y, Todaka T, Sawada M: Mechanism of intracranial rebleeding in moyamoya disease. Clin Neurol Neurosurg 99 (Suppl 2):S187S190, 1997

7. Kawaguchi S, Sakaki T, Morimoto T, Kakizaki T, Kamada
$\mathrm{K}$ : Characteristics of intracranial aneurysms associated with moyamoya disease. A review of 111 cases. Acta Neurochir (Wien) 138:1287-1294, 1996

8. Kim SH, Kwon OK, Jung CK, Kang HS, Oh CW, Han MH, et al: Endovascular treatment of ruptured aneurysms or pseudoaneurysms on the collateral vessels in patients with moyamoya disease. Neurosurgery 65:1000-1004, 2009

9. Kuroda S, Houkin K, Kamiyama H, Abe H: Effects of surgical revascularization on peripheral artery aneurysms in moyamoya disease: report of three cases. Neurosurgery 49:463-468, 2001

10. Lévêque M, McLaughlin N, Laroche M, Bojanowski MW: Endoscopic treatment of distal choroidal artery aneurysm. J Neurosurg 114:116-119, 2011

11. Saeki N, Yamaura A, Hoshi S, Sunami K, Ishige N, Hosoi Y: [Hemorrhagic type of moyamoya disease.] No Shinkei Geka 19:705-712, 1991 (Jpn)

12. Strully KJ: Successful removal of intraventricular aneurysm of the choroidal artery. J Neurosurg 12:317-321, 1955

13. Yuan Z, Woha Z, Weiming X: Intraventricular aneurysms: case reports and review of the literature. Clin Neurol Neurosurg 115:57-64, 2013

14. Zhang L, Xu K, Zhang Y, Wang X, Yu J: Treatment strategies for aneurysms associated with moyamoya disease. Int J Med Sci 12:234-242, 2015

\section{Disclosures}

The authors report no conflict of interest concerning the materials or methods used in this study or the findings specified in this paper.

\section{Author Contributions}

Conception and design: Takeuchi. Acquisition of data: Takeuchi. Analysis and interpretation of data: Takeuchi, Wada. Reviewed submitted version of manuscript: Handa, Wada. Administrative/ technical/material support: Handa, Chu, Wakabayashi. Study supervision: Handa, Chu, Wakabayashi.

\section{Supplemental Information \\ Videos \\ Video 1. https://vimeo.com/260247394.}

\section{Correspondence}

Kazuhito Takeuchi: Nagoya University Graduate School of Medicine, Nagoya City, Aichi, Japan. ktakeuchi@med.nagoya-u.ac.jp. 